\title{
RESEARCH
}

\section{Real-Time, Direct Classification of Nanopore Signals with SquiggleNet}

Yuwei Bao ${ }^{1}$, Jack Wadden ${ }^{1,3}$, John R. Erb-Downward ${ }^{4}$, Piyush Ranjan ${ }^{4}$, Robert P. Dickson ${ }^{4,5,6}$, David Blaauw $^{3}$ and Joshua D. Welch ${ }^{1,2}$

Full list of author information is available at the end of the article

\begin{abstract}
Single-molecule sequencers made by Oxford Nanopore provide results in real time as DNA passes through a nanopore and can eject a molecule after it has been partly sequenced. However, the computational challenge of deciding whether to keep or reject a molecule in real time has limited the application of this capability. We present SquiggleNet, the first deep learning model that can classify nanopore reads directly from their electrical signals. SquiggleNet operates faster than the DNA passes through the pore, allowing real-time classification and read ejection. When given the amount of sequencing data generated in one second, the classifier achieves significantly higher accuracy than base calling followed by sequence alignment. Our approach is also faster and requires an order of magnitude less memory than approaches based on alignment. SquiggleNet distinguished human from bacterial DNA with over $90 \%$ accuracy across test datasets from different flowcells and sample preparations, generalized to unseen species, and identified bacterial species in a human respiratory meta genome sample.
\end{abstract}

Keywords: Deep Learning; Read-Until; Oxford Nanopore; Raw signal; Real-time

\section{Introduction}

Oxford Nanopore sequencers, such as MinION or PromethION, determine the nucleotide sequence of a DNA or RNA molecule by measuring changes in electrical current (called "squiggles") as the molecule translocates through a protein nanopore. This approach is fundamentally different from the widely-used Illumina platform and provides several benefits: the MinION is small, fast, and portable, making it ideal for rapid diagnostics and field work. Because it does not rely upon synchronized nucleotide addition (the heart of the Illumina sequencing-by-synthesis technology), MinION also produces much longer reads (current record around 2Mbp [1]). The changes in electrical current induced by a DNA or RNA molecule depend on the specific chemical properties of the nucleotides, including secondary structure interactions and epigenomic modifications such as methylation. Additionally, the nanopore sequencer can stream the squiggle data to a computer in real time.

The nanopore sequencer can also eject a partially sequenced molecule, a capability referred to as "Read-Until". In principle, this enables targeted sequencing without the need for biochemical enrichment. The Read-Until capability allows selective sequencing of molecules by reversing the voltage across individually selected nanopores, ejecting the unwanted molecules. The unoccupied nanopores can then sequence different molecules of interest. 
Such computational enrichment of target sequences holds great promise for clinical diagnostics and field research, but realizing this potential requires fast and accurate approaches for identifying molecules of interest. For example, identifying pathogenic DNA in a patient lung fluid sample requires bypassing human DNA - which often represents $>99 \%$ of the sequences - to find the pathogen sequences. Biochemical methods for target sequence enrichment, such as PCR [2-5], hybrid capture [6], or CRISPR/Cas9 enrichment [7, 8] require much more time, expertise, and equipment. In contrast, a computational approach to enriching target sequences provides clear savings of time, labor, and cost.

Previous computational approaches for this problem include: a) perform standard base calling followed by sequence alignment as in [9], and b) perform rough base calling to identify and align $k$-mers [10]. The first approach requires significant computing resources - such as a graphics processing unit (GPU) and a large genome index database for the sequence aligner. The second approach also requires a large genome index and multiple CPU cores and can map only non-repetitive references smaller than $\sim 100 \mathrm{Mbp}$. Both approaches are based on sequence alignment and thus are limited by sequencing errors, their reliance on genome indexes, and their inability to capture non-sequence information such as DNA methylation.

To address these limitations, we developed SquiggleNet, the first approach for classifying DNA sequences directly from electrical signals. SquiggleNet is fast, accurate, memory-efficient, and robust to unknown species. It requires only 3000 signals - less than the amount of data generated in one second of sequencing - to classify the species of a DNA molecule with over $90 \%$ accuracy, significantly higher than the best alignment-based methods. The model requires only $304 \mathrm{~KB}$ of RAM and no external reference database. SquiggleNet is faster than or on par with the competitors and can run in real time on a single core of a standard laptop. When tested on a human respiratory metagenome sample with a majority of unseen species, our approach achieves $>90 \%$ overall accuracy.

\section{Results}

\section{SquiggleNet: A Convolutional Neural Network for Classifying Nanopore Signals}

SquiggleNet is a deep neural network that classifies molecules of interest based on statistical patterns in nanopore conductivity, which are often hard for humans to identify by eye, automatically extracted from the input data. The overall workflow for using SquiggleNet to enrich sequences of interest is shown in Figure 1a. The network is first trained to recognize certain classes of sequences, such as human vs. bacterial DNA, using labeled examples. Then, as the nanopore sequencer generates raw electrical signals from a new and unseen sample, SquiggleNet rapidly classifies each molecule to determine whether it is a sequence of interest. Molecules not of interest are ejected from the nanopore, freeing the pore to sequence a different molecule. In contrast, targeted molecules are sequenced to full length and used for downstream analysis.

SquiggleNet (Figure 1b) employs a convolutional architecture, using residual blocks modified from ResNet[11] to perform one-dimensional (time-domain) convolution over squiggles. The architecture consists of four blocks with increasing numbers of channels; each block includes two 1-D ResNet Bottleneck units. Mean 


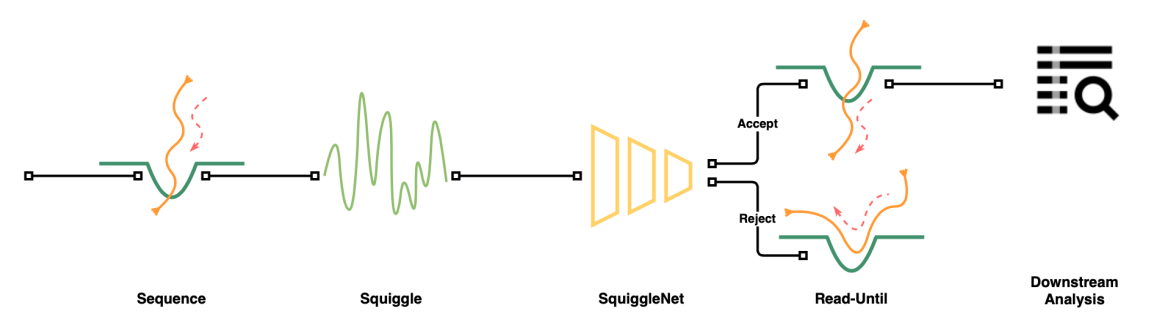

(a) Read-Until Pipeline with SquiggleNet

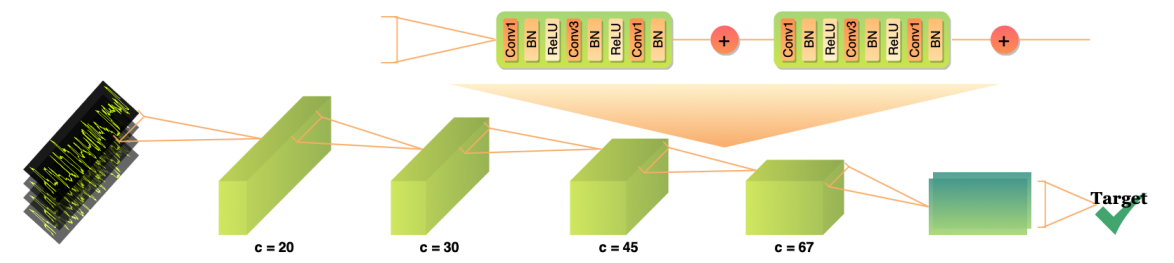

(b) SquiggleNet Architecture

Figure 1: Read-Until Pipeline Overview: (a) A DNA molecule translocates through a nanopore, generating electric signals (squiggles). SquiggleNet rapidly classifies the molecule to determine whether it is a sequence of interest. If the molecule is accepted by the classifier, it is sequenced to full length. Otherwise, the molecule is ejected from the pore, freeing the pore to sequence another molecule. (b) SquiggleNet employs 1D ResNet bottleneck blocks with increasing numbers of filters. We perform average pooling after the last convolutional block and use a final fully connected layer.

pooling followed by a fully-connected layer with softmax activation allows SquiggleNet to classify sequences based on the convolutional filters in the last ResNet block. The final output is a conditional probability on the sequence labels, which is then used to make the final class prediction. We experimented with several other approaches, including a recurrent neural network (RNN) with long short-term memory (LSTM) blocks; gated recurrent units (GRUs); other types of convolutional blocks; a combination of RNN and convolution; different convolutional window sizes; and differing model hyperparameters. However, we found that approaches based on convolution outperformed models using LSTM blocks, suggesting that local features are sufficient for this problem, and long-range time-dependent relationships do not add much information. Convolutional architectures without LSTM blocks are also faster to train. Our final architecture gave the best classification accuracy of any approach we tried and could not be made significantly smaller without sacrificing performance. Additional details about the model architecture and hyperparameter choices can be found in the Method section.

\section{SquiggleNet Accurately Classifies Species Directly from Squiggles}

To test the performance of SquiggleNet, we generated four experimental datasets containing a mixture of human and bacterial DNA. The first dataset, HeLa\&Zymo, 


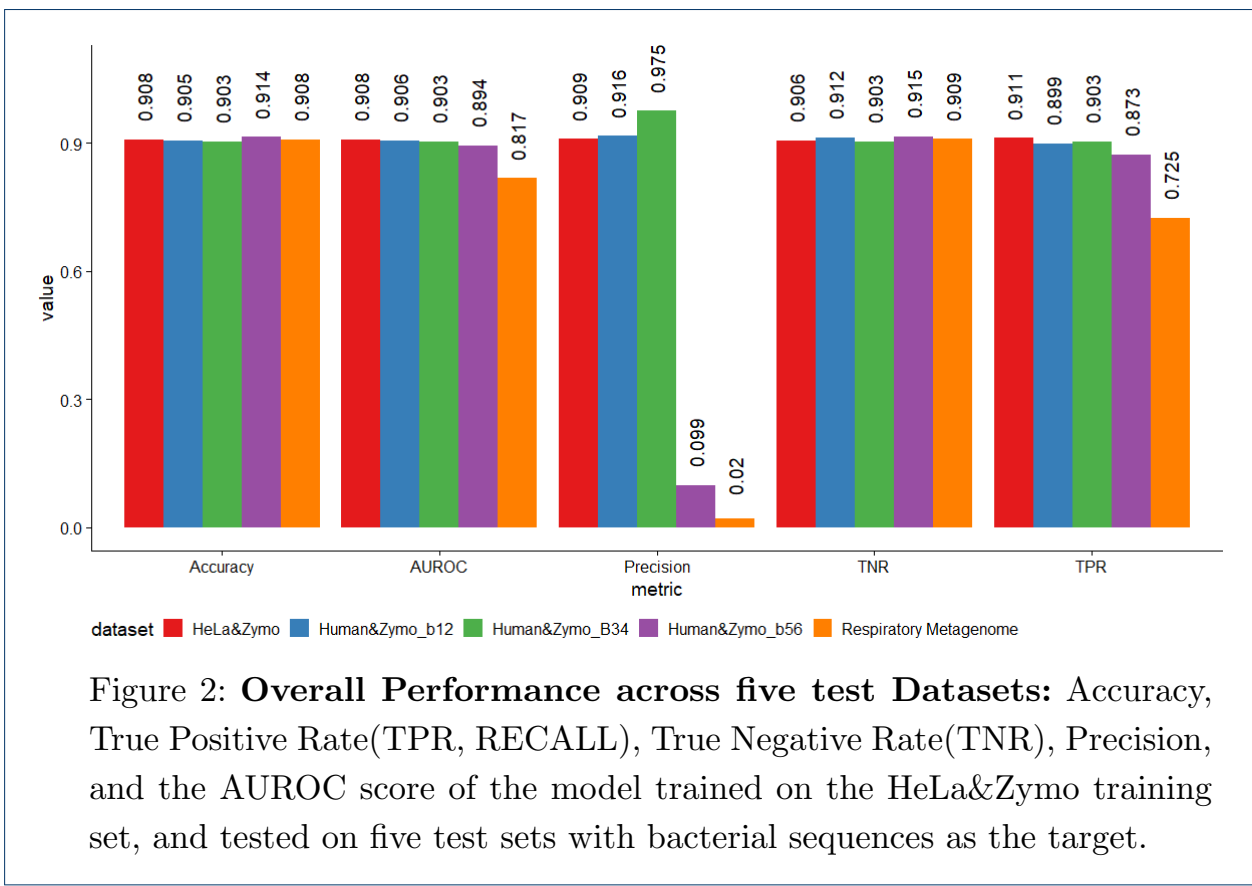

contains 8 bacterial species from the Zymo mixture [12] and HeLa cells. The species labels were obtained through Minimap2 [13] alignment. The other three datasets (Human\&Zymo_b12, Human\&Zymo_b34, and Human\&Zymo_b56) contain a mixture of human NA12878 DNA and DNA from Zymo High Molecular Weight mixture with 7 bacterial species [14]. To avoid systematic error from the alignment algorithms, we obtained reliable ground-truth species labels for these three datasets by attaching a nucleotide sequence barcode to each DNA molecule indicating whether the molecule is from human or bacteria. Note that our direct biochemical labeling strategy allows us to independently assess the accuracy of species determination from base calling followed by read alignment; this is important for our application, since we expect that SquiggleNet may be able to outperform purely sequencebased approaches by leveraging other information from the electrical signals. Further dataset details can be found in the Method section and Supplement.

We trained SquiggleNet using more than two million reads from the first dataset (HeLa\&Zymo), which contains equal proportions of HeLa and bacterial sequences. We used 3000 signals from each read, the equivalent of about 300 nucleotides. We discarded the first 1500 signals of each read, to remove potential pore noise and adapter sequences, which could confound training. Thus SquiggleNet requires a total of 4500 signals, which is equivalent to about 1 second of sequencing time. (The exact time and number of nucleotides depends on the translocation speed, which varies per pore and molecule over the course of the sequencing experiment.) Our best-performing model was trained on the HeLa\&Zymo dataset, which contains the largest number of sequenced reads. This dataset also lacks species-specific barcodes, and we were careful to remove the sequencing adapters and species barcodes before extracting the 3000 signals used for classification (Methods). Thus, there is no way that the classifier could "cheat" by using the barcodes to classify the species. When we instead trained the model on the Human\&Zymo datasets and tested on 
HeLa\&Zymo, the model accuracy was slightly lower, possibly due to the smaller number of training samples (see Supplement and Fig. 8).

Overall, the model classifies each molecule as bacterial or human with over $90 \%$ accuracy across different test datasets using only 3000 signals per read (see Figure 2 ). The classifier generalizes well to different lab preparations, flow cells and proportions of species. For the first three datasets (HeLa\&Zymo, Human\&Zymo_b12, Human\&Zymo_b34), the true positive rates (TPR, also Recall) and the true negative rates (TNR) are all above or around 90\%. The precision and AUROC scores are all about $90 \%$ as well. Even for samples with significantly more human than bacterial DNA (Human\&Zymo_b56 and Respiratory Metagenome), the accuracy and recall both remain high.

To demonstrate that the results are robust to the amount of signal removed from the beginning of the read at test time, we also tested our pre-trained model on the Human\&Zymo_b34 dataset with only the first 1000 signals per read removed. We chose the number 1000 because this is a closer estimation of the adapter length [15], and at test time, we would like to make the decision as soon as possible to enable real-time read selection. When testing the model on sequences with the first 1000 signals removed, the results were nearly identical to those obtained from conservatively removing 1500 signals: $89.35 \%$ accuracy, $90 \%$ true positive rate, and $86.9 \%$ true negative rate. Thus it appears that, as long as the initial pore noise, adaptors, and barcodes are removed from the training sequences, the model is able to make an accurate and fast decision at test time. This robustness also allows flexibility if, for example, different sequencing datasets use sequencing adapters of different lengths.

Remarkably, we find that SquiggleNet achieves significantly higher accuracy from 3000 signals than base calling followed by sequence alignment using the same amount of signal. This result gives crucial context for interpreting the accuracy of our model and suggests that the convolutional filters may detect some nonsequence features that help with species classification, such as chemical modification of nucleotides by methylation. Consistent with this hypothesis, we found that the bacterial and human DNA sequences in our dataset show significant methylation differences (see Supplement for details).

For the Human\&Zymob_56 dataset, the target and non-target sequence ratio is around 99:1. The overall accuracy, TNR, and AUROC score are around 90\%. The TPR (Recall) is closely following, above $87 \%$. The precision, however, is about $1 / 10$ of the other cases. This is due to the extremely low concentration of the targeted sequences (Zymo bacterial species), and the precision calculation is diluted by the overwhelming number of false positive reads. Nevertheless, this is acceptable, since we want to preserve as many targeted reads as possible (high recall) due to the low target read concentration. All true positives and false positives will be sequenced to full length, and thus can be further processed in the downstream analysis. Considering that the Human\&Zymo_b56 dataset has $99 \times$ more non-targeted reads than targeted, whereas only $\sim 10 \times$ more reads were falsely identified as positive compared to the HeLa\&Zymo dataset, this model demonstrated strong ability to filter out non-targeted reads, and has high potential to improve throughput (see below). Overall, the model that was trained on only the HeLa\&Zymo dataset yields high 


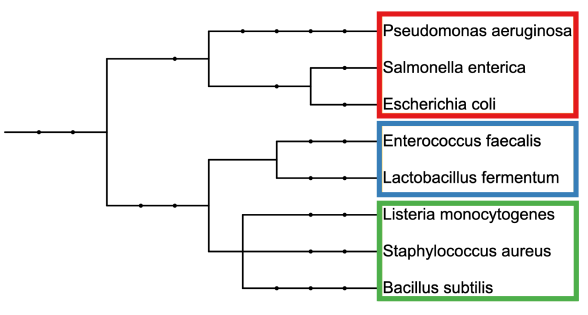

(a) Taxonomy Tree

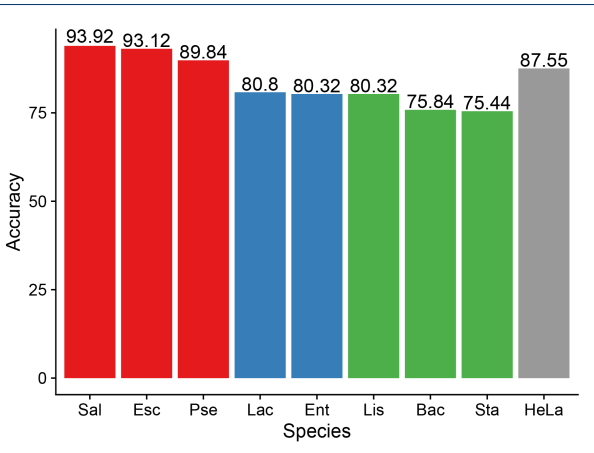

(b) Accuracy Per Species

Figure 3: Taxonomy Tree and Accuracy Per Species: Taxonomy tree for the eight species in our dataset grouped in color and their corresponding accuracy breakdown per species. The orange branch showed highest accuracy per species on average, the yellow group in the middle, and the green group was relatively harder to distinguish from human.

performance across different testing datasets, highlighting the robustness of the model.

Interestingly, SquiggleNet performance varies systematically across bacterial taxa. The network classifies human vs. bacterial DNA with $90 \%$ accuracy, but some bacterial species are easier to distinguish than others. The eight bacterial species in the Zymo mixture are related according to the taxonomy tree shown in Figure 3. The top three species-Pseudomonas aeruginosa (Pse), Salmonella enterica (Sal), and Escherichia coli (Esc)-are gram-negative bacteria and are most easy to identify, while the bottom five species are gram-positive bacteria and are harder to distinguish from human DNA. It is not clear what specific features of the gram-negative bacteria make them easier to identify, but this behavior may be related to species differences in GC-content or the amount of methylation.

\section{SquiggleNet Identifies Species Not Seen During Training}

In real-world applications, patient samples very likely contain species whose genomes are not in the training samples. We thus investigated whether the model can identify unknown species. To do this, we performed a leave-one-out analysis, removing each of the bacterial species separately during training, then putting it back during testing to challenge SquiggleNet's generalization ability. The experiment was conducted on a smaller scale using $400 \mathrm{k}$ and $20 \mathrm{k}$ reads for training and testing, respectively, on the HeLa\&Zymo dataset.

During each training run, we removed one of the eight Zymo bacterial species from the training dataset. We then compared the test accuracy from the classifier trained on seven bacterial species plus human with the performance of the same model on two different testing sets containing the eighth held-out species. The dataset we call Test-Uniform/HeLa includes all eight species (including the one held out during training), evenly distributed, and balanced to contain equal numbers of HeLa and bacterial molecules. The dataset we refer to as Test-One/HeLa includes only the single held-out species and HeLa, in equal proportions. 


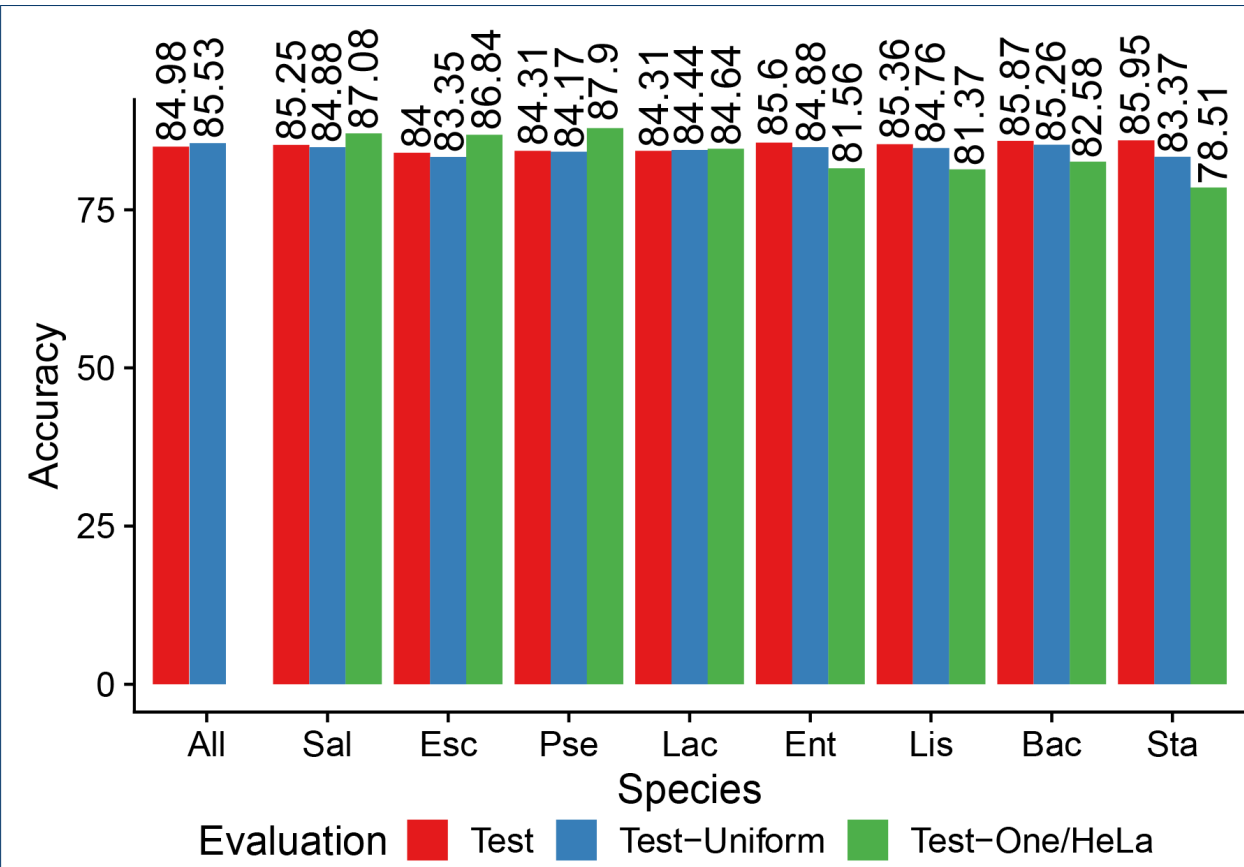

Figure 4: Performance of SquiggleNet on Unseen Species: Each column (except All) is a model trained on a Zymo/HeLa 1:1 mix without the held-out species. For each species, the red bar shows the test accuracy on all species minus the held-out species (Test-Minus); this number provides a baseline against which to compare performance on the held-out species. Blue bars show the accuracy of each trained model on Test-Uniform/HeLa, a test set with all eight Zymo bacterial species included (equal numbers of molecules from each species) and HeLa in a 1:1 ratio. Green bars show the accuracy of each model on Test-One/HeLa, a test set with only the single unseen species and HeLa in a 1:1 ratio.

The unknown species identification results can be found in Figure 4. The orange bars are the test accuracy results without held-out species. The left-most column is the performance of a training run with all 8 bacterial species as a reference for cross-testing run performance comparison. The Test-Uniform/HeLa Diff line is the difference between the Test-Uniform/HeLa accuracy and the test accuracy without held-out species. Similarly, the Test-One/HeLa Diff line is the difference between the Test-Uniform/HeLa accuracy and the test accuracy without held-out species.

Across different runs, the test accuracies, not including held-out species, are around $84 \%-86 \%$. For each Test-Uniform/HeLa experiment, accuracy of classifying the held-out species was $\sim 83 \%-85 \%$, only about $1 \%$ lower compared to when the species was seen during training. This shows that the model was able to accurately identify sequences from bacterial species that were not seen during training. For the Test-One/HeLa experiment, the test performance is more influenced by the taxonomic position of the held-out species. Since the testing datasets only include human DNA and the one species that was held out, we expected performance to drop even more than the previous Test-Uniform/HeLa experiment. However, the test ac- 
curacies of the first three gram-negative bacterial species, Pseudomonas aeruginosa (Pse), Salmonella enterica (Sal), and Escherichia coli (Esc) actually increased by $\sim 1 \%$-4\% compared to their validation accuracies. The remaining four gram-positive species had a minor performance increase or drop within $4 \%$. Staphylococcus aureus had the largest performance drop among all, but the accuracy was still above $72 \%$.

In summary, these two sets of experiments show that even when one species was not seen during training time, SquiggleNet was still able to identify it with high confidence.

\section{SquiggleNet Identifies Bacterial DNA in a Human Respiratory Metagenome Sample}

To further test the generalizability and practicality of SquiggleNet, we tested the best performing model (trained on the Hela\&Zymo dataset) on a dataset collected from several clinical human samples. We collected the data following the procedures in an updated version of [16]. The ground truth labels were obtained using our previously published run-until pipeline[17]. The dataset includes 324526 human reads and 341 bacteria and other (less than 0.6\%), a human:bacterial ratio of 951:1. Some of the dominant bacteria groups include Prevotella (29\%), Neisseria (20\%), and Rothia (11\%). However, only $<3 \%$ of the bacterial species overlap with the training dataset. The full taxonomic composition can be found in Figure 5 and [16].

Even though the model was trained on dataset Hela\&Zymo, it achieved $90.8 \%$ overall accuracy in the Respiratory Metagenome dataset, $72.5 \%$ true positive rate, and $90.9 \%$ true negative rate (Figure 2). The AUROC score is 0.817 . The precision is about $1 / 5$ of that in dataset Human\&Zymo_b56. As with the unbalanced Human\&Zymo dataset, the precision is diluted by the extreme low concentration of bacteria, but the model still achieves high recall-which is critical to retrieve all the bacterial reads for downstream analysis.

The Zymo community of the dataset on which the model was trained has very little overlap $(<3 \%)$ with the bacterial species found in the Respiratory Metagenome dataset. The genome information in this testing dataset was mostly unseen and unknown for the trained model. However, it still achieved a True Positive Rate of $72.5 \%$. This shows that SquiggleNet is able to extract common bacterial genome features and distinguish them from the human genome sequencing raw signals. The generalizability of SquiggleNet significantly increases the potential applications of our method.

\section{SquiggleNet Is More Accurate and Efficient than Previous Approaches}

We next compared the performance and efficiency of SquiggleNet against the current state-of-the-art methods: Guppy+Minimap2 and UNCALLED. This experiment was conducted on dataset Human\&Zymo_b34 with 1:4 Human and Zymo mix. All the analysis was done on a single-usage Intel(R) Xeon(R) CPU E5-2697 v3 @ 2.60GHz machine with a single TITAN Xp GPU.

We benchmarked the running time required to classify 712,000 reads (178 fast5 files with 4000 sequences each and 3000 signals per read, adapters and barcodes removed). SquiggleNet took 806.74 seconds (13 minutes 27 seconds) to finish processing all on GPU (Figure 6). When tested on a $3.5 \mathrm{GHz}$ Dual-Core Intel Core i7 Mac Pro, SquiggleNet finished processing all the files in 2630.58 seconds (43 mins 


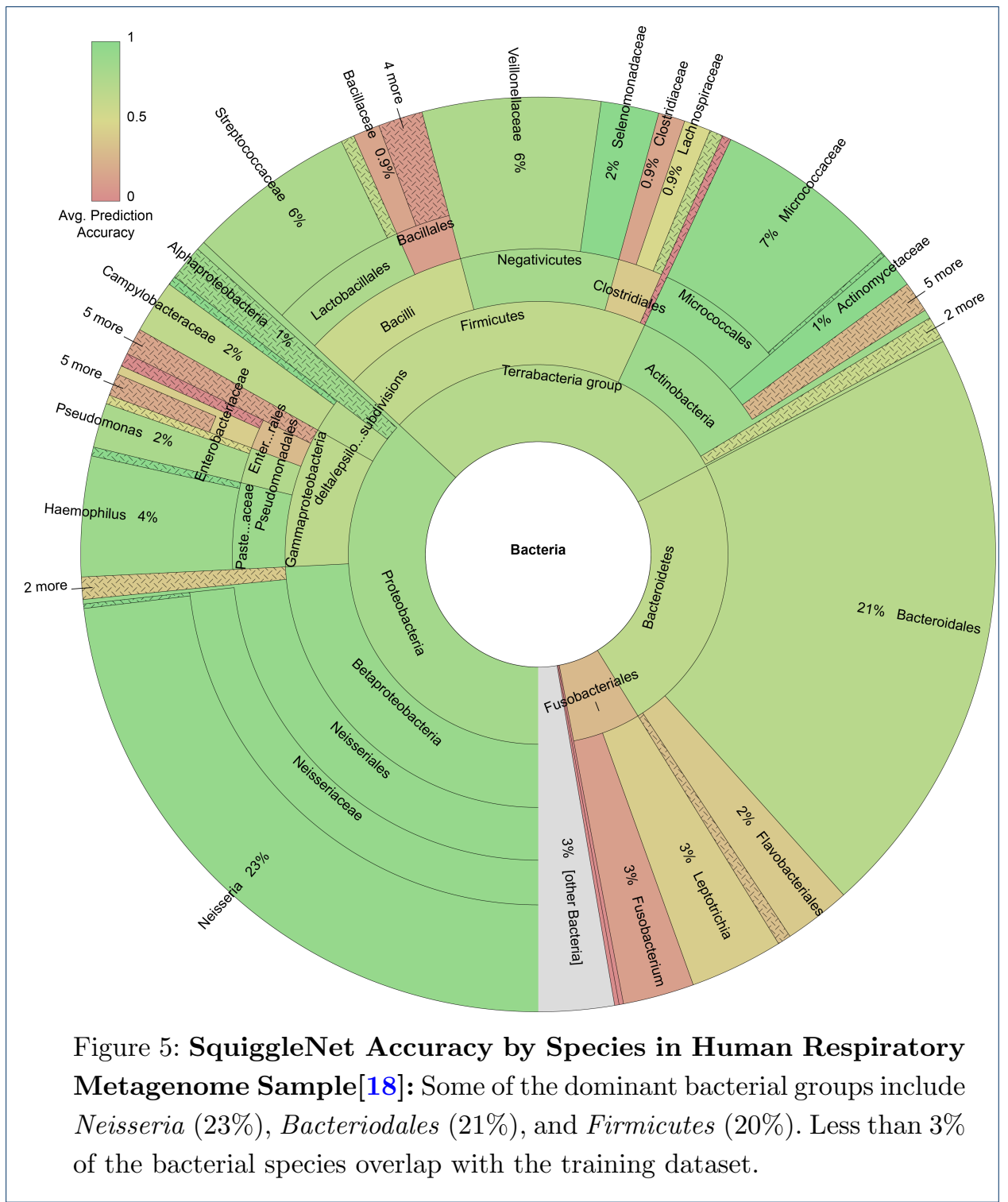

51 seconds). With the Guppy+Minimap2 method [9], sequences were base called using Guppy [19] with 4 callers and 4 runners/GPU, and Minimap2 [13] uses 32 threads for sequence alignment. It took 742.602 seconds (12min 23s) for Guppy to finish basecalling 3000 signals, and another 25.673 seconds for Minimap2 to finish the alignment, about the same amount of time as SquiggleNet. The accuracy of Guppy+Minimap2, however, was 79\%, more than 10\% lower than SquiggleNet. Using the full length of the input sequence increased the accuracy of Guppy+Minimap2 to $91 \%$, but the processing time increased dramatically. With UNCALLED, 32 threads were used to process 3000 signals, 6000 signals, and full-length reads respectively. It took at least 1277.28 seconds (21 min $17 \mathrm{~s})$ to finish the 3000 signals, but the accuracy was below $50 \%$. With longer input length, the accuracy increases to $60 \%$ ( $82 \%$ for full-length), but the full length accuracy is still lower than SquiggleNet with 3000 signals. Meanwhile, the processing time grows drastically as well. 


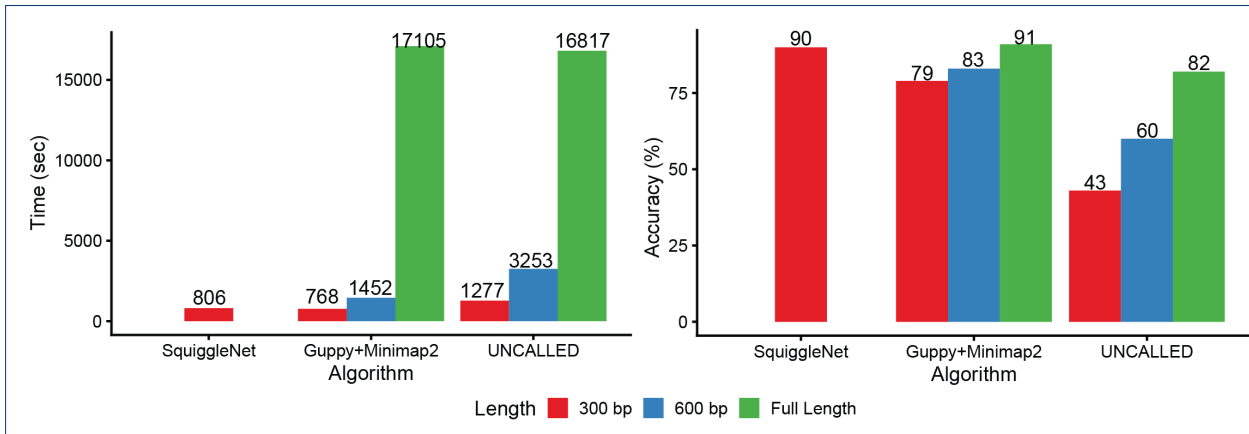

Figure 6: Processing Time and Accuracy Comparison: The processing time of SquiggleNet with $300 \mathrm{bp}$ of input is among the lowest, and yet the accuracy is the highest among the three methods. For the other two alignment-based methods, with longer input length, the processing time grows drastically, whereas the accuracy gain is limited.

SquiggleNet is, therefore, faster and more accurate than either Guppy+Minimap2 or UNCALLED.

We also observed that over $90 \%$ of the SquiggleNet processing time on GPU and over $40 \%$ of the processing time on CPU is spent on loading data from the disk. The actual classification time for a batch of 500 reads is about 0.06 seconds on GPU, and about 0.8 seconds on CPU (one thread). When streaming directly from the nanopore sequencer in real time, this loading time can be largely reduced since it does not require slow disk reads and there is also a lot of room for parallel computing efficiency improvement.

SquiggleNet also offers significant advantages in terms of space requirements, requiring only $304 \mathrm{~KB}$ to store the model parameters. The run-time space is capped by the maximum GPU or CPU memory through adjusting the input batch size. Guppy, however, is a much larger deep-learning model, and the smallest pre-trained option available through Oxford Nanopore Community [19] is 5.5MB. The run-time space is also capped by the maximum GPU memory. On top of that, however, the Guppy+Minimap2 method also requires a customized database for Minimap2 reference. In this experiment, the human and Zymo reference database takes 3.2GB. UNCALLED is currently operational only on CPU. The run-time space is capped by the maximum CPU memory. Similarly, it also takes a reference database to build a Burrows-Wheeler index, which is an extra 3.2GB in this experiment. Therefore, SquiggleNet requires much less space than the other two methods.

\section{SquiggleNet Improves Throughput by Enabling Computationally Targeted Sequencing}

To assess the potential improvement in sequencing throughput that SquiggleNet could provide, we developed a mathematical model to compare the total number of

Table 1: Method Requirement Comparison

\begin{tabular}{ccccc} 
& SquiggleNet & SquiggleNet & Guppy+Minimap2 & UNCALLED \\
\hline Equipment(t=thread) & GPU & CPU(t=1) & GPU + CPU(t=32) & CPU $(\mathrm{t}=32)$ \\
$\begin{array}{c}\text { Space Requirement } \downarrow \\
\text { Model Size } \downarrow\end{array}$ & $\leq$ GPU Max & $\leq$ Mem Max & $\leq$ GPU Max $+3.2 \mathrm{~GB}$ & $\leq$ Mem Max $+3.2 \mathrm{~GB}$ \\
$\mathbf{3 0 4} \mathbf{~ K B}$ & $\mathbf{3 0 4} \mathbf{~ K B}$ & $5.5 / 40 / 116 \mathrm{MB}$ &
\end{tabular}


base pairs and total sequencing time needed to obtain a certain number of targeted sequences with and without SquiggleNet.
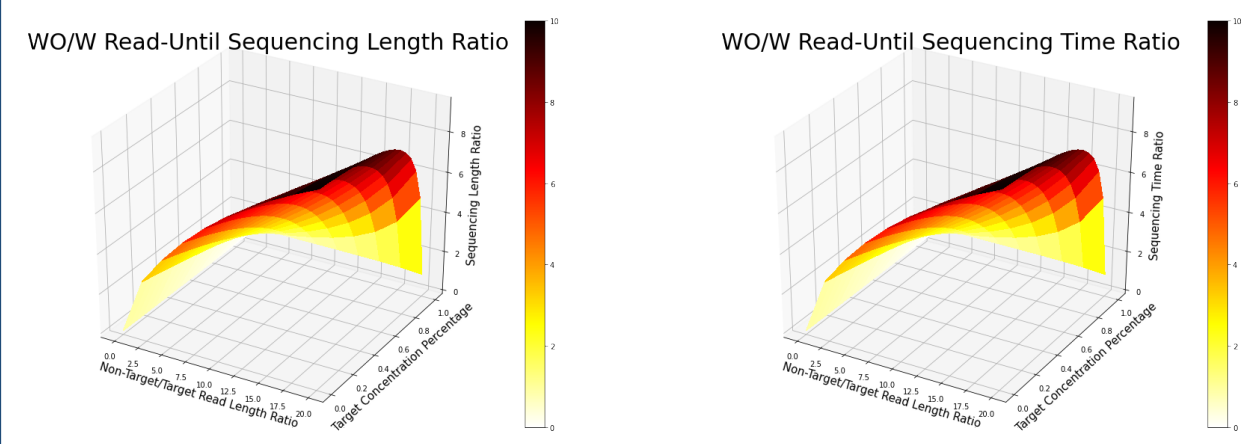

Figure 7: Throughput and Sequencing Time Comparison Without/With Read-Until: When the average non-target read length is about 20 times longer than the target read length, and sample contains over 90\% non-target reads, a normal sequencing pipeline would have to sequence $\sim 10$ times more base pairs (left) than Read-Until pipeline with SquiggleNet to achieve a fixed number of targeted reads. The ratio is about 10 for the required sequencing time as well (right).

The detailed derivation of the model can be found in the Supplement. The most influential hyper-parameters include average target sequence length $\bar{z}$, average nontarget sequence length $\bar{h}$, and target sequence concentration $c$. Several other tunable hyper-parameters, including the waiting time to eject one molecule and begin sequencing another; the total number of active pores in a flow cell; the sequencing speed; and the total number of targeted sequences, did not significantly influence the predicted increase in throughput (see Supplement). We chose the values for these less influential parameters based on the empirical time requirements and accuracy of SquiggleNet and the sample means from the real sequencing data.

In Figure 7, we picked the average non-target/target sequence length ratio as one axis, and the target sequence concentration as the other axis to demonstrate the total number of base pairs (left) and total sequencing time (right) that a regular nanopore sequencing pipeline would require, compared to those of a pipeline with Read-Until + SquiggleNet, in order to obtain a fixed number of targeted reads. Based on the properties of our sequencing datasets, we set average sequencing speed to be 450 base pair per second, and the total number of active pores in a flow cell to be 500. We also used the following parameters based on SquiggleNet's empirical performance: $\mathrm{TPR}=0.9, \mathrm{TNR}=0.9$, sequencing time $=1 \mathrm{~s}$, and classification decision time $=0.8 \mathrm{~s}$ (time required for SquiggleNet on CPU).

We show the predicted gains in throughput and sequencing time for a range of the most important hyperparameters (Figure 7). When the average non-target read length is about 20 times longer than the target read length, and the sample contains over $90 \%$ non-target sequences, it would take a nanopore sequencing pipeline $\sim 10$ times longer than Read-Until pipeline with SquiggleNet to achieve a fixed number of targeted reads. The regular nanopore sequencing pipeline would also have to 
sequence $\sim 10$ times more base pairs than the Read-Until pipeline. Even if we set these parameters much more pessimistically, the model still predicts about a 5fold gain in throughput and time. These numbers are also in the same ballpark as the $4.5 \times$ enrichment reported in the UNCALLED paper[10], supporting the plausibility of our mathematical model. We therefore conclude that Read-Until with SquiggleNet holds great promise to improve target read throughput, saving sequencing time and resources.

\section{Discussion}

The success of our approach suggests that the raw sequencing signals generated by nanopore sequencing contain rich information for identifying target sequences from background sequences. Such features could include different DNA modification patterns, codon frequencies, GC content, or even DNA shape or RNA secondary structure. Furthermore, because these features are primarily local in nature, only a small amount of sequencing signal is required. In contrast, approaches that rely only on sequence information alone require much more signal (more sequencing time), are susceptible to base-calling errors, and do not leverage non-sequence information.

We also note that different reads go through the pores at different speeds. Future work could also include an event detector and a scaler into the classifier, which may further improve performance. Additionally, the squiggles from different MinION flow cells show systematic run-to-run differences. Thus, the data preprocessing and normalization procedures that we employed are crucial for generalizing across datasets.

We tested the capability of our model to enrich the bacterial DNA in the presence of more abundant human DNA. Human and bacterial DNA are significantly different, which makes this classification task feasible. However, we also envision using SquiggleNet to enrich target sequences in other contexts, trained or fine-tuned on user-specific datasets, such as specific regions of the human genome. We also anticipate that SquiggleNet will be useful for distinguishing viral DNA or RNA sequences from host molecules within infected cells. Rapidly identifying targeted sequences could be helpful in numerous clinical settings, including cancer diagnosis, respiratory pathogen identification, and Coronavirus testing.

\section{Methods}

\begin{tabular}{cccccc} 
& \multicolumn{2}{c}{ Table 2: Datasets Description } & \\
Name & Ratio & Train & Validation & Test & Note \\
\hline HeLa\&Zymo & $1: 1$ & $2.4 \mathrm{M}$ & $40 \mathrm{k}$ & $54 \mathrm{k}$ & \\
Human\&Zymo_b12 & $1: 1$ & $1.72 \mathrm{M}$ & $40 \mathrm{k}$ & $44 \mathrm{k}$ & Barcode 1 \& 2 \\
Human\&Zymo_b34 & $1: 4$ & & & $224 \mathrm{k}$ & Barcode 3 \& 4 \\
Human\&Zymo_b56 & $99: 1$ & & & $100 \mathrm{k}$ & Barcode 5 \& 6 \\
Respiratory Metagenome & $951: 1$ & & & 324867 & Real Patient Samples
\end{tabular}

\section{Data Collection}

We generated five datasets using a MinION sequencer (Table 2) for this paper. The first four datasets used the standard Rapid Sequencing Kit (SQK-RAD004) protocol on a FLO-106D MinION Flow Cell. The HeLa\&Zymo dataset used the 
ZymoBIOMICS Microbial Community DNA Standard, and Datasets 2-4 used the ZymoBIOMICS HMW DNA Standard with different barcodes specified in Table 2. Details about the dataset composition can be found in the Supplement. The Respiratory Metagenome data collection method can be found in [16].

Because base calling and sequence alignment of noisy nanopore reads can result in systematic errors and is not a completely reliable source of ground truth, we used barcodes to label the sequences in the three Human\&Zymo datasets as either bacteria or human before mixing them together. The labels obtained in this way thus represent reliable ground truth.

Each extracted signal read was normalized with fast5 scaling and offset. All reads were also normalized using Z-scored median absolute deviation. The extreme signal values with a modified z-score larger than 3.5 were replaced by the average of closest neighbors.

\section{Model Architecture}

SquiggleNet is a 1D-ResNet-based binary classifier (Figure 1). The first layer of 1D-CNN is comparable to the first layer of Guppy[19], but with significantly fewer channels (20 instead of 512). After that, there are four layers of 1D-ResNet, and each layer includes two BottleNeck blocks. The number of channels for each layer increases by a factor of 1.5, and each BottleNeck block decreases the string size with a stride of 2 . We perform average pooling after the final convolutional layer, followed by a fully connected layer. We also experimented with other different architectures (see Supplement).

\section{Training and Evaluation}

Our best-performing model was trained on the HeLa\&Zymo dataset with binary cross-entropy loss. The dataset was split into training, validation, and testing sets. The Human\&Zymo_b12, Human\&Zymo_b34, Human\&Zymo_b56, and Respiratory Metagenome datasets were used to assemble testing sets for the best-performing model.

As a separate analysis, we also trained on the Human\&Zymo datasets and tested on the HeLa\&Zymo datasets. The performance of this model was nearly identical but slightly worse than the model trained on HeLa\&Zymo (see Supplement for details).

The Adam optimizer was used for over 6 epochs on each dataset, with a learning rate of $1 \mathrm{e}-3$ and batch size of 1000 . The model was initialized using Kaiming initialization in fan-out mode. Batch normalization was conducted within each Bottleneck block.

Evaluation metrics include overall accuracy, true positive rate (TPR, Recall), true negative rate (TNR), Precision, and area under receiver operating characteristic curve (AUROC) when running the model on different test datasets. Speed and memory comparisons were performed on the same Intel(R) Xeon(R) CPU E5-2697 v3@2.60GHz machine with a single TITAN Xp GPU. 


\section{Supplement}

\section{Data Collection}

We generated five datasets using a MinION sequencer (Table 2) for this paper. The first four datasets used the standard Rapid Sequencing Kit (SQK-RAD004) protocol on FLO-106D MinION Flow Cell. The HeLa\&Zymo dataset used the ZymoBIOMICS Microbial Community DNA Standard, and Datasets 2-4 used the ZymoBIOMICS HMW DNA Standard with different barcodes specified in Table 2. The Respiratory Metagenome data collection method is described in [16].

The theoretical composition of the ZymoBIOMICS Microbial Community DNA Standard [12] includes 8 types of bacteria with $12 \%$ each of: Listeria monocytogenes (Lis), Pseudomonas aeruginosa (Pse), Bacillus subtilis (Bac), Escherichia coli (Esc), Salmonella enterica (Sal), Lactobacillus fermentum (Lac), Enterococcus faecalis (Ent), Staphylococcus aureus (Sta); and 2 types of fungi with (2\% each): Saccharomyces cerevisiae and Cryptococcus neoformans. The theoretical composition of the ZymoBIOMICS HMW DNA Standard [14] includes 7 types of bacteria with $14 \%$ each of: Listeria monocytogenes, Pseudomonas aeruginosa, Bacillus subtilis, Escherichia coli, Salmonella enterica, Enterococcus faecalis, Staphylococcus aureus; and 1 type of fungus (2\%): Saccharomyces cerevisiae.

The HeLa\&Zymo dataset contains 200ng of HeLa DNA and 200ng of ZymoBIOMICS Microbial Community DNA Standard computed by volume and listed concentrations. The sequenced samples were basecalled using Guppy v3.6.1 and aligned with reference downloaded from NCBI using Minimap2. We assigned the species of these sequences using the Minimap2 alignment because no species barcodes were used in this dataset. Due to the limited fungus sequences, only the bacterial sequences were kept for training, validation, and testing. The first 1500 signals for each read (about 150bp of sequence on average) were removed to avoid adapter sequences and signal instability.

The assembly of the Human\&Zymo datasets each started with 400ng of Human NA12878 DNA and 400ng of ZymoBIOMICS HMW DNA Standard. They were barcoded using the SQK-RBK004 barcodes 1 and 2, 3 and 4, 5 and 6 respectively as indicated in the table. Then each dataset was generated using $200 \mathrm{ng}$ of barcoded NA12878 (by volume) and 200ng of HMW Zymo (by volume) with a total of 400ng pooled in 10ul. For the Human\&Zymo datasets, the extracted samples were basecalled using Guppy v3.6.1 with "-barcode_kits SQK-RBK004" specification to separate the reads and identify the barcodes. The barcodes were then used to identify the species of each read. The first 2000 (about 200bp worth of sequences on average) signals were removed to avoid barcode overfitting and signal instability. Based on the adapter, barcode and barcode flanking sequence description from the Nanopore Community [20, 21], 2000 signals should be more than enough to remove all above.

Each extracted signal read was normalized with fast5 scaling and offset. All reads were also normalized using Z-scored median absolute deviation. The extreme signal values with a modified z-score larger than 3.5 were replaced by the average of closest neighbors. 
bioRxiv preprint doi: https://doi.org/10.1101/2021.01.15.426907; this version posted January 20, 2021. The copyright holder for this preprint (which was not certified by peer review) is the author/funder, who has granted bioRxiv a license to display the preprint in perpetuity. It is made

\begin{tabular}{|c|c|c|c|c|c|c|c|}
\hline Model & Dataset & Layers & Channels & Window Size & Stride & Val Acc & Test Acc \\
\hline \multirow[t]{11}{*}{ "VGG_1000 } & $1 \mathrm{M} / 20 \mathrm{k} / 20 \mathrm{k}$ & 2 & [20] & {$[19,5]$} & [6] & 65.58 & 63.94 \\
\hline & & 2 & [100] & {$[19,5]$} & [6] & 67.06 & 65.85 \\
\hline & & 4 & {$[20,50]$} & {$[19,5,6]$} & {$[3,2]$} & 70.93 & 69.33 \\
\hline & & 4 & {$[40,60,100,100]$} & {$[19,5,6]$} & {$[3,2]$} & 71.78 & 70.61 \\
\hline & & 6 & {$[20,50,75]$} & {$[19,5,6,3]$} & {$[3,1,1]$} & 70.42 & 69.36 \\
\hline & & 6 & {$[40,75,100]$} & {$[19,5,6,3]$} & {$[3,1,1]$} & 71.61 & 69.99 \\
\hline & & 8 & {$[20,20,20,20]$} & {$[20,6,6,3,3]$} & {$[2,1,1,1]$} & 72.21 & 71.16 \\
\hline & & 8 & {$[30,30,30,30]$} & {$[20,6,6,3,3]$} & {$[2,1,1,1]$} & 74.21 & 72.60 \\
\hline & & 8 & {$[50,50,50,50]$} & {$[20,6,6,3,3]$} & {$[2,1,1,1]$} & 76.63 & 75.99 \\
\hline & & 8 & {$[20,50,75,75]$} & {$[19,5,6,3,3]$} & {$[3,1,1,1]$} & 72.95 & 70.68 \\
\hline & & 10 & {$[10,20,40,80,80]$} & {$[5,5,5,5,5]$} & {$[1,1,1,1,2]$} & 77.46 & 76.73 \\
\hline Model & Dataset & & & Block & Layer & Val Acc & Test Acc \\
\hline \multirow[t]{8}{*}{ ResNet_1000 } & $200 \mathrm{k} / 10 \mathrm{k} / 10 \mathrm{k}$ & & & BottleNeck & {$[2,2,2,2]$} & 72.99 & 72.04 \\
\hline & & & & BottleNeck & {$[1,1,1,1]$} & 72.73 & 71.67 \\
\hline & & & & BottleNeck & {$[1,1,1,1,1]$} & 72.12 & 70.98 \\
\hline & & & & BottleNeck & {$[2,2,1,1]$} & 72.91 & 71.07 \\
\hline & & & & BottleNeck & {$[1,1,2,2]$} & 72.81 & 71.45 \\
\hline & $1 \mathrm{M} / 20 \mathrm{k} / 20 \mathrm{k}$ & & & Basic & {$[2,2,2,2]$} & 80.21 & 79.74 \\
\hline & & & & BottleNeck & {$[2,2,2,2]$} & 78.85 & 78.71 \\
\hline & & & & +GroupChannel & {$[2,2,2,2]$} & 77.95 & 77.56 \\
\hline+ LSTM & & & & BottleNeck & {$[2,2,2,2]$} & 80.28 & 79.56 \\
\hline +AdamW & & & & BottleNeck & {$[2,2,2,2]$} & 79.90 & 79.88 \\
\hline \multirow[t]{2}{*}{ Bonito_1000 } & $1 \mathrm{M} / 20 \mathrm{k} / 20 \mathrm{k}$ & & & $1^{*} 2$ & {$[8,8,16,16]$} & 72.44 & 72.63 \\
\hline & & & & $2 * 2$ & {$[8,8,8,16,16]$} & 73.49 & 74.36 \\
\hline Model & Dataset & CNN Dim & Window Size & Hidden size & Layer & Bidirectional & Val Acc \\
\hline \multirow[t]{2}{*}{ CNN_3000+LSTM } & $1 \mathrm{M} / 20 \mathrm{k} / 20 \mathrm{k}$ & 50 & 19 & 50 & 2 & Yes & 64.538 \\
\hline & & 75 & 19 & 75 & 4 & Yes & 64.238 \\
\hline \multirow[t]{2}{*}{ CNN_3000+GRU } & & 50 & 19 & 50 & 2 & Yes & 64.398 \\
\hline & & 75 & 19 & 75 & 4 & Yes & 64.692 \\
\hline
\end{tabular}

Table 3: Selected Model Architecture Experiment Results and Hyperparameter Tuning Performance Report 


\section{Model Architecture Experiments and Hyperparameter Tuning}

In this section, we describe some of the additional models and hyperparameter settings that we explored while developing SquiggleNet. Table 3 summarizes these results. Most of the model architectures were trained on the HeLa\&Zymo dataset using 1000 signals per sequence, after removing the first 1500 signals. We used this shorter input length and a subset of the training dataset to enable more rapid model testing. We then re-trained some of the more promising models with 3000 signals per read. Each model was trained for 2 to 5 epochs until the validation accuracy started to plateau or show signs of overfitting.

The experiments based on the VGG_1000 architecture indicated that a model with more layers and more channels could generally offer better performance. However, the lack of shortcut skips in the architecture limited the possible depth of the VGG models.

We thus next experimented with a ResNet model with different numbers of layers, different numbers of blocks in each layer, different block types, and several other modifications. We found that increasing the number of blocks in each layer boosted performance somewhat, but increasing the number of layers did not necessarily improve the performance. Increasing the size of the training dataset boosted the model performance by $\sim 8 \%$ according to Table 3 . We introduced a layer of LSTM in the middle of our ResNet model, and this increased performance by a small margin, but took much longer to train.

Recurrent Neural Network (RNN) models such as LSTM and GRU gave little advantage over CNN based models and also required significantly more resources to train. For the last set of selected experiments presented in Table 3, we used 3000 signals per read, introduced one layer of CNN with window size 19 to reduce the input size, and followed with various RNN architectures. The performance slowly increased from random guessing $(50 \%)$, but plateaued around $65 \%$. This could indicate that local features extracted by convolution provide sufficient information for classification, and long-range dependencies extracted by the recurrent network only help by a small amount.

Other models we tried include: a down-sized Bonito [22, 23], stacks of LSTM layers with variational window sizes, different hyperparameter settings, and different training datasets. After full consideration of model size, speed, performance, and training time, we settled on the best performing model architecture to perform the main experiments in the paper.

\section{Comparison of Models Trained on HeLa\&Zymo and Human\&Zymo}

In addition to the best-performing model trained on the HeLa\&Zymo dataset, we conducted the same set of test experiments on a model trained on the HymanZymo_b12 dataset. This dataset consists of a 1:1 mix of human DNA and Zymo HMW DNA, as described in Supplement Section Data Collection. We tested on all five datasets with different sample preparation, flow cell, different sample component, and ratio. The performance can be found in Figure 8. The overall performance is almost identical with Figure 2, but slightly worse. This may be because the HymanZymo_b12 dataset contains less training data than the HeLa\&Zymo dataset. Overall, this analysis indicates that the model achieves high accuracy whether 


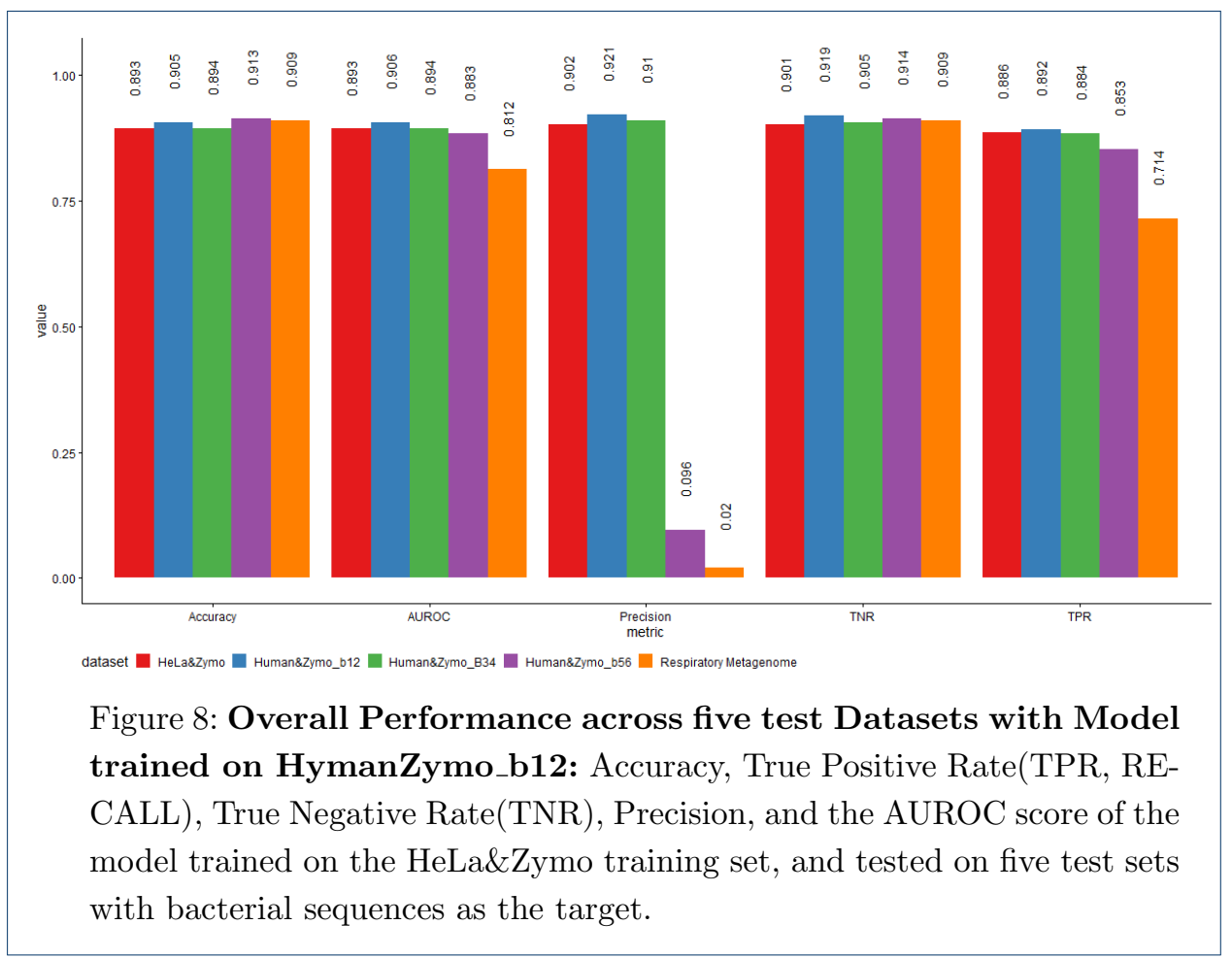

trained on HeLa DNA (which is a highly mutated cancer cell line) or GM12878 DNA (which should look more like healthy human DNA), when tested on the other type of data.

\section{Comparison Experiment Method Details}

The performance and efficiency comparison experiment was conducted on dataset Human\&Zymo_b34 with 1:4 Human and Zymo mix. For a fair read-in and writeout time comparison across the platforms, all the reads were pre-truncated into 3000 signals, 6000 signals, and full length, after removing the first 2000 signals for adapters and barcode. Any reads shorter than the minimum input requirements were discarded. The ground truth labels for each read were obtained by the SQKRBK004 barcode 3 and 4, basecalled by Guppy v3.6.1. Any reads that were labeled as other barcodes or not labeled were discarded.

SquiggleNet was tested on 1) a single-usage Intel(R) Xeon(R) CPU E5-2697v3 @ $2.60 \mathrm{GHz}$ machine with a single TITAN Xp GPU with batch size one (fast5 file, about 4000 sequences each), and on 2) a single-usage $3.5 \mathrm{GHz}$ Dual-Core Intel Core i7Mac Pro with single thread. Over $90 \%$ of the compute time on GPU was spent on file read-in and write-out (over $40 \%$ of the time on CPU).

When testing the Guppy+Minimap2 method, we used 4 callers and 4 runners per GPU for Guppy, and 32 threads for Minimap2 sequence alignment. Indexes for both human and Zymo community reference genome were pre-generated. The test datasets with 3000 signals per read, 6000 signals per read, and full length reads were basecalled by Guppy and then aligned using Minimap2 with the pre-generated index. The resulting read IDs were cross-referenced with the ground truth labels for barcode 3 and 4 to calculate the overall accuracy. 
When testing the UNCALLED method, we used 32 threads to process 3000 signals, 6000 signals, and full-length reads. BWA index was pre-generated for the Zymo community reference genome. Unfortunately, UNCALLED struggles to map repetitive references longer than $100 \mathrm{Mbp}$, such as the human genome. Therefore, we had to treat all the reads that weren't classified as Zymo to be human reads as an approximation for the performance computation. The resulting read IDs were cross referenced with the ground truth labels for barcode 3 and 4 , to calculate the overall accuracy.

\section{DNA Methylation Differences Between Human and Bacterial DNA}

\begin{tabular}{ccc} 
& Avg mAs/KB & Avg mCs/KB \\
\hline Bacterial & 1.72 & 3.53 \\
Human & 0.01 & 16.27
\end{tabular}

Table 4: Average number of methylated $\mathrm{A}(\mathrm{mA})$ and methylated $\mathrm{C}(\mathrm{mC})$ nucleotides in the first 1000 nucleotides of human and bacterial DNA sequences

Because SquiggleNet achieves higher classification accuracy than base calling followed by sequence alignment (given 3000 signals), we hypothesized that the neural network may be using other information besides just nucleotide sequence. One possible such source of information is methylation. We therefore investigated whether there are systematic methylation differences between the human and bacterial DNA sequences that the classifier could detect.

To do this, we used Guppy v3.6.1 to output the methylation probability for each sequence in Dataset Hyman\&Zymo_b34. For each base pair with greater than zero percent chance to be an A or $\mathrm{C}$ (the only nucleotides for which Guppy will predict methylation), we computed the most likely base call: A, methylated A, C, or methylated C. We then calculated the total and average numbers of methylated As and methylated Cs in the first 1000 bases of both human and bacterial sequences.

As shown in Table 4, bacterial sequences contain on average about 2 methylated A nucleotides and 4 methylated $\mathrm{C}$ nucleotides per kilobase, whereas the human sequences contain on average 0 methylated As and 16 methylated Cs per kilobase. This suggests that methylation is indeed a possible feature that could help to classify sequences as bacterial or human. We leave further investigation of the features learned by our model, such as with neural network interpretation approaches, for future work.

\section{Throughput Estimation Model}

The throughput estimation model computes the total amount of time $t$ and the number of base pairs $l$ needed to achieve $x$ targeted reads, and compare the values with and without read-until.

The estimation model assumes the average targeted read length to be $\bar{z}$, and the average non-targeted read length to be $\bar{h}$. It assumes the total number of pores alive to be constant $k$ throughout the sequencing run. It also assumes all the live pores are actively sequencing or in the process of getting a new read. All the other parameter assumptions can be found in table 5 .

Without Read-Until, the total sequencing time $t_{w o}$ and the total number of base pairs $l_{w o}$ needed for $x$ targeted sequences are: 
Table 5: Throughput Estimation Model Parameters

\begin{tabular}{lcc} 
Description & Symbol & Default Value \\
\hline Total sequencing time & $t$ & \\
Total base pair sequenced & $l$ & \\
Number of targeted reads & $x$ & 1000 \\
Number of total reads & $n=x / a$ & \\
Number of pores alive & $k$ & 400 \\
Average sequencing speed & $v$ & $450 \mathrm{bp} / \mathrm{s}$ \\
Average read length: target & $\bar{z}$ & $40000 \mathrm{bp}$ \\
Average read length: non-target & $\bar{h}$ & $40000 \mathrm{bp}$ \\
Concentration: target & $a$ & 0.1 \\
Concentration: non-target & $b=1-a$ & 0.9 \\
Classifier TPR & $p_{1}$ & $90 \%$ \\
Classifier TNR & $p_{2}$ & $90 \%$ \\
Time: attract a new read & $t_{0}$ & $0.01 \mathrm{~s}$ \\
Time: initial sequencing & $t_{1}$ & $1 \mathrm{~s}$ \\
Time: decision time & $t_{2}$ & $0.8 \mathrm{~s}$ \\
Time: reject a read & $t_{3}$ & $0.01 \mathrm{~s}$
\end{tabular}

$$
\begin{gathered}
l_{w o}=[\bar{z} a+\bar{h} b] n \\
t_{w o}=\left[\left(t_{0}+\bar{z} / v\right) a+\left(t_{0}+\bar{h} / v\right) b\right] \cdot n / k
\end{gathered}
$$

With Read-Until, the total sequencing time $t_{r u}$ and the total number of base pairs $l_{r u}$ needed for $x$ targeted sequences are:

$$
l_{r u}=\left[\left(t_{1} v+t_{2} v\right)\left(\left(1-p_{1}\right) a+b p_{2}\right)+\bar{z} a p_{1}+\bar{h} b\left(1-p_{2}\right)\right] n
$$

$t_{r u}=\left[\left(t_{0}+t_{1}+t_{2}+t_{3}\right) b p_{2}+\left(t_{0}+t_{1}+t_{2}+t_{3}\right) a\left(1-p_{1}\right)+\left(t_{0}+\bar{z} / v\right) a p_{1}+\left(t_{0}+\bar{h} / v\right) b\left(1-p_{2}\right)\right] \cdot n / k$

Several parameters cancel out and thus do not affect the sequencing length ratio $l_{w o} / l_{r u}$ or the sequencing time ratio $t_{w o} / t_{r u}$. These parameters include: the number of targeted reads $x$, the number of total reads $n$, and the number of active nanopores $k$. Several hyperparameters were set to default values based on SquiggleNet's statistics and the sample means from multiple sequencing experiments. These include average sequencing speed $(v=450 \mathrm{bp} / \mathrm{s})$, classification TPR $\left(p_{1}=90 \%\right)$, classification TNR $\left(p_{2}=90 \%\right)$, initial sequencing time $\left(t_{1}=1 s\right)$, and decision time $\left(t_{1}=0.8\right)$. The average read length for target and non-target reads are both defaulted as $40000 \mathrm{bp}$. The target concentration is defaulted to be 10\%, and non-target read concentration to be $90 \%$.

We also investigated how $t_{0}$, the time to attract a new read, or $t_{3}$, affect the throughput ratio. We varied these two parameters from 0 to 1 second and plotted the resulting throughput ratio for both sequencing length and sequencing time (Figure 9). Neither parameter significantly affected the throughput ratio. 
bioRxiv preprint doi: https://doi.org/10.1101/2021.01.15.426907; this version posted January 20, 2021. The copyright holder for this preprint (which was not certified by peer review) is the author/funder, who has granted bioRxiv a license to display the preprint in perpetuity. It is made Bao et al.

Page 20 of 22
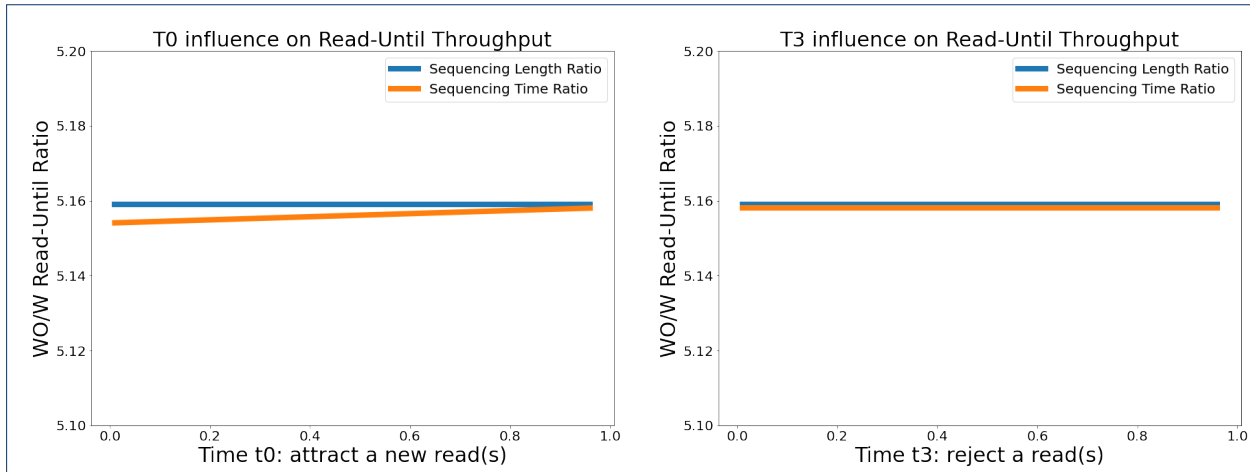

Figure 9: The influence of to and t3 on Read-Until Throughput: As t0 and t3 change from 0 to 1 second, the sequencing time and length ratio (without/with read-until) are not effected significantly. 
bioRxiv preprint doi: https://doi.org/10.1101/2021.01.15.426907; this version posted January 20, 2021. The copyright holder for this preprint (which was not certified by peer review) is the author/funder, who has granted bioRxiv a license to display the preprint in perpetuity. It is made

\section{Availability of data and materials}

We have uploaded our data to SRA (SRP296988). The code can be found in our Github repository:

https://github.com/welch-lab/SquiggleNet.

Competing interests

The authors declare no competing interests.

Funding

JDW wishes to acknowledge support from NIH grants R01 Al149669-01 and R01 HG010883-01.

\section{Author's contributions}

YB and JDW conceived the idea of SquiggleNet. JRE initially suggested the idea of direct classification on Nanopore signals for read-until speed up. YB designed and implemented the model and analyzed the data. JW generated datasets 1 to 4 . JRE, PR and RPD provided the human-pathogen dataset and Figure 5. YB and JDW wrote the paper. JRE and DB offered suggestions and advice. All authors read and approved the final manuscript.

Acknowledgements

We thank members of the CELab and Prof. Jenna Wiens for their helpful suggestions and advice during this project.

Author details

1 Department of Computer Science and Engineering, University of Michigan, 48109 Ann Arbor, Michigan, USA

2 Department of Computational Medicine and Bioinformatics, University of Michigan, 48109 Ann Arbor, Michigan, USA

3 Department of Electrical and Computer Engineering, University of Michigan, 48109 Ann Arbor, Michigan, USA

4 Division of Pulmonary and Critical Care Medicine, Department of Internal Medicine, University of Michigan

Medical School, 48109 Ann Arbor, Michigan, USA

5 Department of Microbiology and Immunology, University of Michigan Medical School, 48109 Ann Arbor,

Michigan, USA

. 6 Michigan Center for Integrative Research in Critical Care, 48109 Ann Arbor, Michigan, USA.

\section{References}

1. Oxford Nanopore: Minion. https://nanoporetech.com/products/minion

2. Gnirke, A., Melnikov, A., Maguire, J., Rogov, P., LeProust, E.M., Brockman, W., Fennell, T., Giannoukos, G., Fisher, S., Russ, C., Gabriel, S., Jaffe, D.B., Lander, E.S., Nusbaum, C.: Solution hybrid selection with ultra-long oligonucleotides for massively parallel targeted sequencing. Nature Biotechnology 27(2), 182-189 (2009). doi:10.1038/nbt.1523

3. Kozarewa, I., Armisen, J., Gardner, A.F., Slatko, B.E., Hendrickson, C.L.: Overview of target enrichment strategies (1934-3647 (Electronic))

4. Rand, A.C., Jain, M., Eizenga, J.M., Musselman-Brown, A., Olsen, H.E., Akeson, M., Paten, B.: Mapping dna methylation with high-throughput nanopore sequencing. Nature Methods 14(4), 411-413 (2017). doi: $10.1038 /$ nmeth. 4189

5. Simpson, J.T., Workman, R.E., Zuzarte, P.C., David, M., Dursi, L.J., Timp, W.: Detecting dna cytosine methylation using nanopore sequencing. Nature Methods 14(4), 407-410 (2017). doi:10.1038/nmeth.4184

6. Charalampous, T., Kay, G.L., Richardson, H., Aydin, A., Baldan, R., Jeanes, C., Rae, D., Grundy, S., Turner, D.J., Wain, J., Leggett, R.M., Livermore, D.M., O'Grady, J.: Nanopore metagenomics enables rapid clinical diagnosis of bacterial lower respiratory infection. Nature Biotechnology 37(7), 783-792 (2019). doi:10.1038/s41587-019-0156-5

7. Gilpatrick, T., Lee, I., Graham, J.E., Raimondeau, E., Bowen, R., Heron, A., Sedlazeck, F.J., Timp, W.: Targeted nanopore sequencing with cas 9 for studies of methylation, structural variants, and mutations. bioRxiv, 604173 (2019). doi:10.1101/604173

8. Gu, W., Crawford, E.D., O'Donovan, B.D., Wilson, M.R., Chow, E.D., Retallack, H., DeRisi, J.L.: Depletion of abundant sequences by hybridization (dash): using cas9 to remove unwanted high-abundance species in sequencing libraries and molecular counting applications. Genome Biology 17(1), 41 (2016). doi:10.1186/s13059-016-0904-5

9. Payne, A., Holmes, N., Clarke, T., Munro, R., Debebe, B.J., Loose, M.: Readfish Enables Targeted Nanopore Sequencing of Gigabase-sized Genomes. doi:10.1038/s41587-020-00746-x

10. Kovaka, S., Fan, Y., Ni, B., Timp, W., Schatz, M.C.: Targeted Nanopore Sequencing by Real-time Mapping of Raw Electrical Signal with UNCALLED. doi:10.1038/s41587-020-0731-9

11. He, K., Zhang, X., Ren, S., Sun, J.: Deep Residual Learning for Image Recognition (2015). 1512.03385

12. ZymoBIOMICS Microbial Community DNA Standard. https://www.zymoresearch.com/collections/zymobiomics-microbial-community-standards/products/ zymobiomics-microbial-community-dna-standard

13. Li, H.: Minimap2: pairwise alignment for nucleotide sequences. Bioinformatics 34(18), 3094-3100 (2018). doi:10.1093/bioinformatics/bty191. https://academic.oup.com/bioinformatics/article-pdf/34/18/3094/25731859/bty191.pdf

14. ZymoBIOMICS HMW DNA Standard. https://www.zymoresearch.com/collections/ zymobiomics-microbial-community-standards/products/zymobiomics-hmw-dna-standard

15. Oxford Nanopore Technologies, M.L.: Real-Time Selective Sequencing on the MinION. Youtube. https: //www . youtube. com/watch?v=34sWScdYyYQ\&t=303s\&ab_channel=0xfordNanoporeTechnologies

16. O'Dwyer, D.N., Ashley, S.L., Gurczynski, S.J., Xia, M., Wilke, C., Falkowski, N.R., Norman, K.C., Arnold, K.B., Huffnagle, G.B., Salisbury, M.L., Han, M.K., Flaherty, K.R., White, E.S., Martinez, F.J., Erb-Downward, J.R., Murray, S., Moore, B.B., Dickson, R.P.: Lung microbiota contribute to pulmonary inflammation and 
bioRxiv preprint doi: https://doi.org/10.1101/2021.01.15.426907; this version posted January 20, 2021. The copyright holder for this preprint (which was not certified by peer review) is the author/funder, who has granted bioRxiv a license to display the preprint in perpetuity. It is made available under aCC-BY-NC-ND 4.0 International license.

Bao et al.

Page 22 of 22

disease progression in pulmonary fibrosis. American journal of respiratory and critical care medicine 199(9), 1127-1138 (2019). doi:10.1164/rccm.201809-16500C

17. Pendleton, K.M., Erb-Downward, J.R., Bao, Y., Branton, W.R., Falkowski, N.R., Newton, D.A.-O., Huffnagle, G.A.-O., Dickson, R.A.-O.: Rapid pathogen identification in bacterial pneumonia using real-time metagenomics (1535-4970 (Electronic))

18. Ondov, B.D., Bergman, N.H., Phillippy, A.M.: Interactive metagenomic visualization in a web browser. BMC Bioinformatics 12(385), 1471-2105 (2011)

19. Oxford Nanopore: Guppy.

https://community.nanoporetech.com/protocols/Guppy-protocol/v/GPB_2003_v1_revT_14Dec2018

20. Oxford Nanopore: Barcoding Kits. https://community.nanoporetech.com/technical_documents/ chemistry-technical-document/v/chtd_500_v1_revw_07jul2016/barcoding-kits

21. Oxford Nanopore: Rapid Sequencing Kit Family. https://community.nanoporetech.com/technical_documents/chemistry-technical-document/v/chtd 500_v1_revw_07jul2016/rapid-sequencing-kit-family

22. Kriman, S., Beliaev, S., Ginsburg, B., Huang, J., Kuchaiev, O., Lavrukhin, V., Leary, R., Li, J., Zhang, Y.: QuartzNet: Deep Automatic Speech Recognition with 1D Time-Channel Separable Convolutions (2019). 1910.10261

23. Bonito. https://github.com/nanoporetech/bonito 